\title{
Investigating the Residual Stress Distribution in Selective Laser Melting Produced Ti-6Al-4V using Neutron Diffraction
}

\author{
L.S. Anderson ${ }^{1, a}$, A.M. Venter ${ }^{2, b}$, B. Vrancken ${ }^{3, c}$, D. Marais ${ }^{2}$, J. van Humbeeck ${ }^{3, d}$ \\ and T.H. Becker ${ }^{1, \mathrm{e}^{*}}$ \\ ${ }^{1}$ Department of Mechanical and Mechatronic Engineering, Stellenbosch University, South Africa \\ ${ }^{2}$ Research and Development Division, South African Nuclear Energy Corporation, South Africa \\ ${ }^{3}$ Department of Materials Engineering, University of Leuven, Belgium \\ alanderson@sun.ac.za, bandrew.venter@necsa.co.za, cbey.vrancken@kuleuven.be, \\ djan.vanhumbeeck@kuleuven.be, ${ }^{\mathrm{t}}$ tbecker@sun.ac.za
}

\begin{abstract}
Keywords: Neutron Diffraction, Selective Laser Melting, Residual Stress, Additive Manufacturing, Ti-6Al-4V
\end{abstract}

\begin{abstract}
The Selective Laser Melting (SLM) process makes rapid manufacture of both prototype and structural components possible for a variety of metals. However, high residual stresses are inherent to the building process and can pose a number of problems; including part distortion, cracking and a reduction in the components' mechanical strength and fatigue life. Exact stress distributions through the part volume are often not known as the methods commonly used to measure residual stresses are surface or near surface stresses. As such, the influence of build parameters, residual stresses on resultant SLM-produced part integrity is not understood. Neutron diffraction allows for the measurement of residual stress through the volume of a part using measured lattice strains, thereby providing a tool to gain insight into the SLM process and the formation of residual stresses. In this work, neutron diffraction was used to determine the distribution of residual stress in a set of rectangular SLM-produced Ti-6Al-4V samples. Results show that an increase in layer thickness reduces the stress gradients in the part. There is also evidence that changing the exposure strategy can prevent stresses from developing along a preferential axis, making a more homogeneous stress field.
\end{abstract}

\section{Introduction}

Ti-6Al-4V is an $\alpha / \beta$ titanium-based alloy that contains, by weight, $4 \% \mathrm{Al}$ and $6 \% \mathrm{~V}$ as its alloying elements [1]. Ti-6Al-4V is used extensively in the medical industry as well as in the aerospace industry in high strength-to-weight ratio components [2]. In recent years it has become a material of interest for use in Additive Manufacturing (AM) processes, in particular Selective Laser Melting (SLM) [1]. SLM is categorised as a powder bed fusion AM technology that selectively melts powder into a solid three-dimensional part using a high-powered laser source. SLM is capable of producing highly dense, net shape components [3]. As shown in Fig. 1, thin layers of atomized fine metal powder are evenly distributed using a coating mechanism from a powder hopper onto a baseplate that move along the vertical (z) axis. This takes place inside a chamber containing a tightly controlled atmosphere of inert gas, commonly argon. Once each layer has been distributed, each slice of the part geometry is fused by selectively melting the powder using a high-power laser beam, typically between $20 \mathrm{~W}$ and $1 \mathrm{~kW}$ [4]. The laser beam is directed (in the $\mathrm{x}$ and $\mathrm{y}$ axis directions) with two high frequency scanning mirrors. The laser energy is intense enough to permit full melting of the particles to form solid metal [5]. The process is repeated layer by layer until the part is complete. 


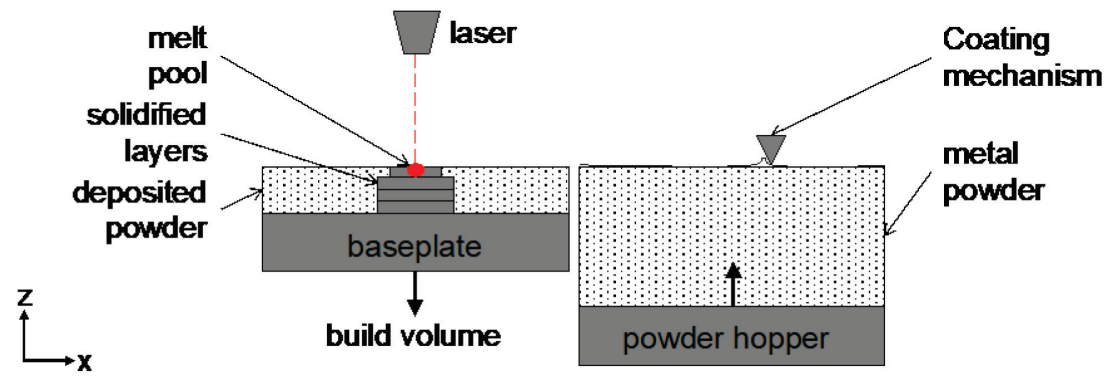

Fig 1. SLM process that selectively melts powder into a solid three-dimensional part using a high-powered laser source. Note the co-ordinate system where the $x$ and $y$ (out of the page) directions are parallel to the baseplate and the $z$ direction is the build direction.

It is this melting and solidification cycle that results in the formation of residual stresses [6]. The primary mechanism is driven by a temperature gradient mechanism, which is a result of rapid, localized, heating that occurs at the impingement point of the laser on the material [7]. Large thermal gradients form due to the slow conduction of heat away from the melt pool, which result in a mismatch in the thermal expansion experienced by the molten material and the solidified material surrounding it [7]. The thermal expansion of the molten material is thus constrained by the solidified material surrounding it, resulting in compressive stress in the solid material. If the expansion is sufficient, the compressive stress in the constraining solid material exceeds its yield strength, causing it to deform plastically. Upon cooling of the molten region, a secondary mechanism occurs, whereby the thermal contraction of the molten region during the state change from liquid to solid is constrained by the plastically deformed solid material surrounding it. This constraint induces tensile stress in the newly solidified region and as a result the top build layer tends to develop a net tensile stress. A number of techniques, including X-ray diffraction, hole drilling and the contour method have been used to determine the influence of build parameters on residual stress in SLM Ti-6Al-4V, however, no investigation has been performed on the influence of build parameters on the stress distribution through the full volume of an SLM-produced Ti-6Al-4V part.

This study is aimed at investigating the through-volume residual stress distribution in a set of SLM-produced Ti-6Al-4V samples using neutron diffraction (ND). A brief introduction and background to the work is given first, followed by the details of the ND based stress measurements. The results obtained, and their relevance are presented and discussed.

\section{Materials and methodology}

The Ti-6Al-4V powder was acquired from 3D Systems Layerwise. A size distribution analysis showed that the particles ranged in size from $5 \mu \mathrm{m}$ to $50 \mu \mathrm{m}$, with a median size of $\mathrm{d}_{50}=$ $34.43 \mu \mathrm{m}$. Rectangular samples with dimensions $20 \times 20 \times 10 \mathrm{~mm}^{3}$ in $\mathrm{x}, \mathrm{y}$ and $\mathrm{z}$ directions (as shown in Fig. 2(a)) were built at KU Leuven's Department of Metallurgy and Materials Engineering using an in-house developed SLM machine. The coordinate system used for the production of the samples is the same as that used in ASTM F2921, where the layers are deposited in the $x-y$ plane and the sample is grown along the z-axis. All samples were wire cut from the base plate after manufacture, before residual stress measurements commenced.

The test matrix consisted of four samples; two layer thicknesses of 30 and $90 \mu \mathrm{m}$, and three exposure strategies, where the laser path would follow a parallel ([0]), perpendicular $([0 / 90])$ and with a $30^{\circ}([0 / 30 / 60 / \ldots])$ rotation to the previous layers laser path and are summarised in Table 1. 
(a) Investigation plane

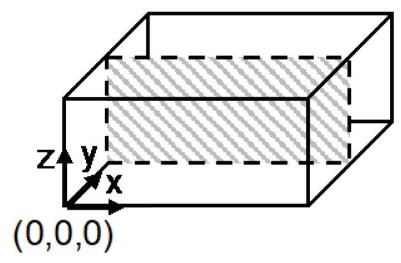

(b) Measurement grid

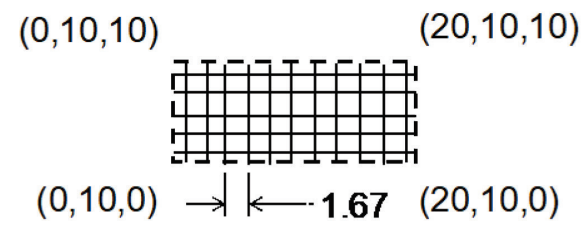

(c) Measurement point

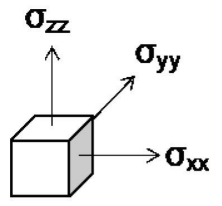

Fig. 2 Depiction of the ND measurements points taken: (a) investigation plane, (b) $11 x 5$ measurement grid of $1.67 \mathrm{~mm}$ spacing (in $x$ and $z$ ) and (c) calculated stress tensor at a specific grid point. All dimensions given in $\mathrm{mm}$.

Table 1. Common and sample specific SLM build parameters used.

Common parameters

\begin{tabular}{|c|c|c|}
\hline Laser power [W] & Laser speed [mm/min] & Hatch spacing $[\mu \mathrm{m}]$ \\
\hline 250 & 1800 & 75 \\
\hline \multicolumn{2}{|c|}{ Sample-specific parameters } \\
\hline Sample name & Exposure strategy $^{1}\left[{ }^{\circ}\right]$ & Layer thickness $[\mu \mathrm{m}]$ \\
\hline $1-30$ & {$[0]$} & 30 \\
\hline $2-30$ & {$[0 / 90]$} & 30 \\
\hline $2-90$ & {$[0 / 90]$} & 90 \\
\hline $3-30$ & {$[0 / 30 / 60 \ldots]$} & 30 \\
\hline
\end{tabular}

Archimedes test according to ASTM of the samples revealed an average density of minimum of $99.42 \%$ (sample 2-90) and a maximum of $99.58 \%$ (sample 3-30).

ND measurements were performed at the South African Nuclear Energy Corporation (Necsa) using the MPISI neutron strain scanning instrument. Measurements were taken through the centre of the sample, along an investigation plane (normal to the $\mathrm{y}$-axis in the $\mathrm{x}$ and $\mathrm{z}$ direction as shown in Fig. 2a). This investigation plane was discretized into a $5 \times 11$ grid of measurement points approximately $1.67 \mathrm{~mm}$ apart (as shown in Fig. 2b). Strain measured for the three orthogonal directions matching the sample coordinate system were taken at each grid point using the parameters detailed in Table 2 to provide normal stress measurements in $\sigma_{\mathrm{xx}}$ and $\sigma_{\mathrm{yy}}$, as shown in Fig. 2c. This measurement strategy was chosen on the assumption that the stress distribution would be approximately symmetrical about the $\mathrm{x}-\mathrm{z}$ plane due to the symmetry of the exposure strategies and sample geometry. A $2 \times 2 \times 2 \mathrm{~mm}^{3}$ ND analysis region was chosen to allow sufficient accuracy in ND measurements, as well as sufficient spatial resolution.

Table 2. Parameters used for ND strain mapping.

\begin{tabular}{|c|c|c|c|c|}
\hline $\begin{array}{c}\text { analysis region } \\
{\left[\mathrm{mm}^{3}\right]}\end{array}$ & $\begin{array}{c}\text { Psi tilt } \\
{\left[{ }^{\circ}\right]}\end{array}$ & $\begin{array}{c}\text { Bragg angle } \\
{\left[{ }^{\circ}\right]}\end{array}$ & $\begin{array}{c}\text { Miller indices of } \\
\text { planes [hkl] }\end{array}$ & $\begin{array}{c}\text { Average scan time } \\
\text { per sample [hrs] }\end{array}$ \\
\hline $2 \times 2 \times 2$ & 90 & 77.6 & $(103)$ & 202 \\
\hline
\end{tabular}

The zero-stress lattice spacing was determined for each sample by assuming that the normal stress component in the z-direction $\left(\sigma_{z z}\right)$ is zero. This was based on initial tests that indicated that the stress in the normal z-direction is approximately zero i.e. that the material was in a biaxial state of stress. The reference spacing $d_{0}$ was calculated using Eq. 1, based on which the $\sigma_{x x}$ and $\sigma_{y y}$ could be computed using Eq. 2 [8]. 


$$
d_{0}=\frac{d_{z}\left(S_{2}+2 S_{1}\right)}{S_{1}}-\frac{s_{1}\left(d_{x}+d_{y}\right)}{S_{2}}
$$

In Eq. $1, d_{0}$ is the calculated stress-free reference spacing, $S_{1}$ and $S_{2}$ are the diffraction elastic constants for $\{103\}$ planes, and $d_{x}, d_{y}$ and $d_{z}$ are the measured plane spacings along the $x, y$, and $z$ directions, respectively. In Eq. 2, $\sigma_{i i}$ represents the normal stress components for $\sigma_{x x}$ and $\sigma_{y y}$ in units of $\mathrm{MPa}[8]$.

$$
\sigma_{i i}=\frac{d_{i}-d_{z}}{d_{0}} * \frac{1 \cdot 10^{6}}{S_{2}}, \quad \mathrm{i}=(\mathrm{x}, \mathrm{y})
$$

It is noted that in order to achieve a full stress tensor, six independent directions are needed to obtain the six stress components. This would result in testing durations becoming impractically long. The current setup of two stress directions and 55 sampling points requires about 10 days per sample. The average measurement error was $26.2 \mathrm{MPa}$ for all samples. Respective stress gradients between adjacent points were calculated by numerically differentiating the obtained stress components and relative positions using central differencing scheme. This was done using Matlab's (ver. 2017a) gradient function.

\section{Results and discussion}

Stress distribution: Stress contour plots of $\sigma_{\mathrm{xx}}$ and $\sigma_{\mathrm{yy}}$ are shown for sample 2-30 in Fig. 3a. Stress values indicate an approximately parabolic shaped stress distribution in $\mathrm{x}$ and $\mathrm{z}$. Furthermore, a symmetrical stress distribution is observed, as well as symmetry between $\sigma_{\mathrm{xx}}$ and $\sigma_{\text {yy }}$. Sample 3-30 showed a similar symmetry (not shown), however, sample 1-30 showed significantly lower stresses in the $\sigma_{\mathrm{yy}}$ direction, i.e. perpendicular to the laser path (not shown). This is due to the contraction of the heated material being more severely constrained in the laser path direction. Exposure strategies $[0 / 90]$ and $[0,30,60, \ldots]$, for which the laser path direction is alternated between each layer, tend to provide an equal stress state.

Influence of layer thickness: The influence of layer thickness is observed by comparing samples 2-30 and 2-90. The measured stresses are shown in Fig. 3b on the left at three build heights and respective stress gradients on the right. The comparison shows that a thinner layer thickness exhibits higher stress magnitudes (in both tension and compression), as well as steeper stress gradients. This result is consistent with previous research that showed that both the stress magnitude and gradient are a function of the number of build layers [7]. The data suggests that a larger layer thickness can offer advantages, from a residual stress point-of-view, by allowing for lower tensile and compressive stresses at the sample surface and centre respectively.

Influence of exposure strategy: The influence of the exposure strategy is considered in Fig. 3c by comparing samples 130, 230 and 330. Shown on the left are respective stress values at a build height of $\mathrm{z}=5 \mathrm{~mm}$ and on the right the stress biaxiallity ratio, defined as $\sigma_{\mathrm{xx}} / \sigma_{\mathrm{yy}}$. Note that a value near one indicates equal stresses in $\sigma_{\mathrm{xx}}$ and $\sigma_{\mathrm{yy}}$, whereas a value near 0.3 (Poisson's ratio) indicates a uniaxial stress state. The data shows a clear effect of the exposure strategy and stress homogeneity. Literature suggests that the exposure strategy influences the directionality of the stress distribution in the $x-y$ plane. This directionality of stress is most evident when comparing sample 1-30 to sample 3-30, which shows a considerable variation in stress between the sample centre and surface. Both exposure strategies for sample 2-30 and 3-30 tilt the laser path and thereby the principal axes, resulting in a more homogeneous stress field. This is evident in the right figure (Fig. 3c) where sample 2-30 and 3-30 show a near biaxial stress state in the middle of the sample. 
(a)
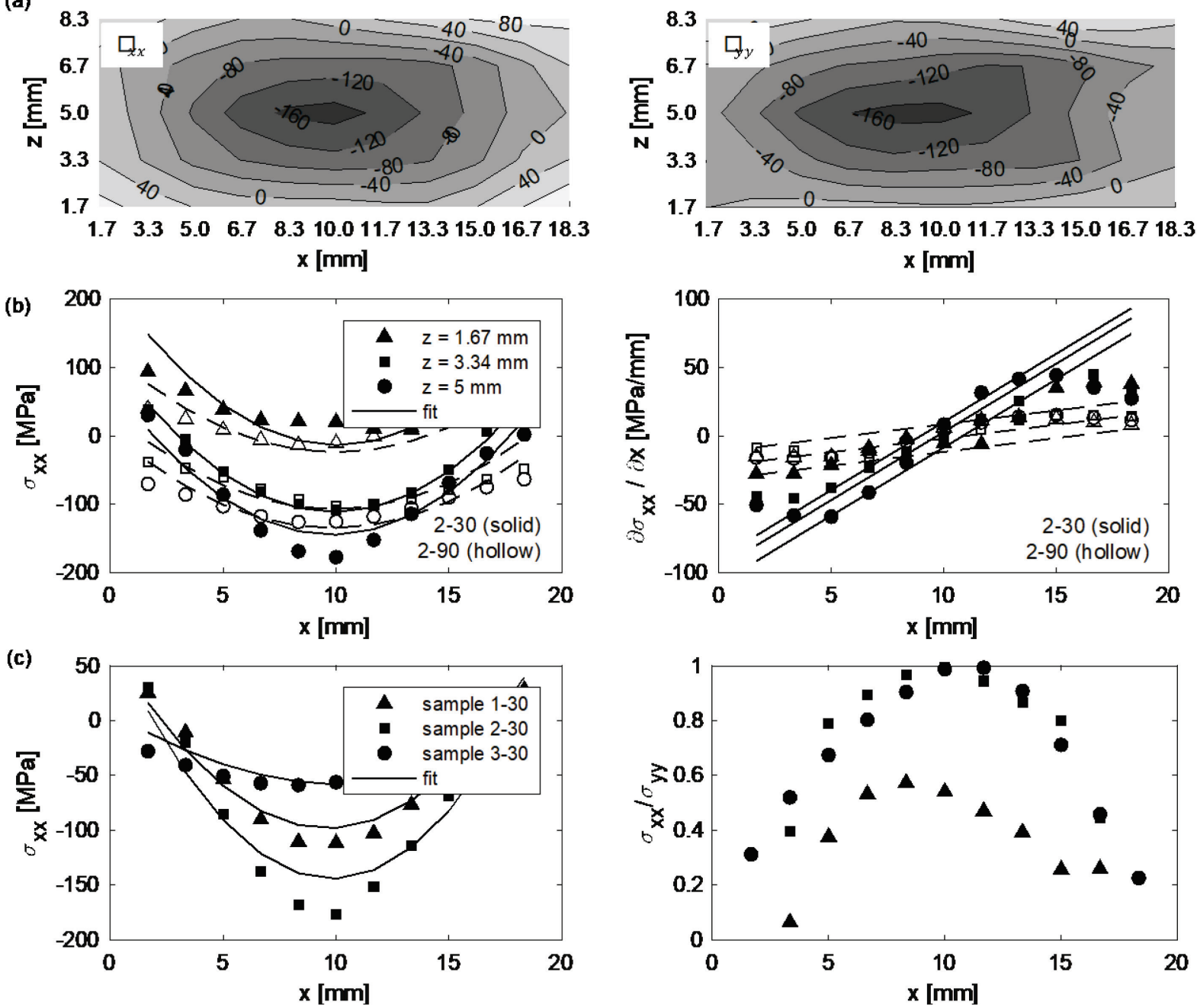

Fig. 3 (a) residual stresses for 2-30 in $\sigma_{x x}$ (left) and $\sigma_{y y}$ (right). (b) comparing stress values (left) and gradients (right) between a 30 and 90 um layer thickness. (c) comparing stress values (left) and biaxiallity ratio (right) between the [0], [0/90] and [0, 30,60, ...] exposure strategies.

Measurement discussion points: The use of ND for stress measurements in SLM produced Ti$6 \mathrm{Al}-4 \mathrm{~V}$ uses analysis regions that are large compared to the build layers. For example, a sample built with $30 \mu \mathrm{m}$ layer thickness results in 67 layers per analysis region. This means that the stresses are averaged over a large number of layers and as such the results obtained indicate an average stress value and not a maximum or minimum value.

Studies have shown that residual stresses decrease in magnitude by up to $80 \%$ in the first $1.5 \mathrm{~mm}$ from the surface of SLM-produced parts [9]. Since this is less than the size of the analysis region, peak stresses cannot be obtained. Furthermore, high systematic errors that can occur if an analysis region is defined so that it protrudes from the sample and therefore surface and near-surface stress measurements cannot be performed. This loss of stress information is of vital importance as it is this near surface stress which most greatly affects the performance of SLM components.

One of the main limiting factors in this investigation was scan times. As indicated in Table 2, a single sample required 10 days of testing. Ideally, full mapping of the stress values in the 
samples should consider the full stress tensor consisting of 6 stress components [8]. This was not feasible, and the study assumed zero stress state in $\sigma_{\mathrm{zz}}$ based to initial testing undertaken. This stress state assumption is not a true representation of the state of stress within the samples, meaning that the use of Eq. 1 and Eq. 2 may not provide the correct stress values. However, the results do provide insight into the dependence of layer thickness and exposure strategy on the stress distribution. It is argued that the study provides sufficient evidence to motivate for exposure strategies that significantly alter the laser path between layers, as well as for larger layer thicknesses.

\section{Conclusion}

This study measured the bulk stress distribution in SLM-produced Ti-6Al-4V samples using ND. The study considered layer thickness and exposure strategy of identically sized rectangular samples. The following observations were made:

- Approximately parabolic shaped stress distributions were observed for rectangular samples.

- Stress magnitudes and gradients can be seen as a function of build layer thickness. By increasing the layer thickness, the stress magnitudes and gradients are significantly reduced.

- The exposure strategy used has a direct influence on the homogeneity of the stress state. A unidirectional exposure strategy results in preferentially orientated stress in the direction of the scan vector. By increasing the number of scan vector orientations, the stress components become more homogeneous and a biaxial stress state is achieved.

\section{References}

[1] M. Simonelli, Microstructure Evolution and Mechanical Properties of Selective Laser Melted Ti-6Al-4V, Loughborough University, 2014.

[2] H. J. Rack and J. I. Qazi, Titanium alloys for biomedical applications, Mater. Sci. Eng. C, 26(8) (2006) 1269-1277. https://doi.org/10.1016/j.msec.2005.08.032

[3] B. Ferrar, L. Mullen, E. Jones, R. Stamp and C. J. Sutcliffe, Gas flow effects on selective laser melting (SLM) manufacturing performance, J. Mater. Process. Tech. 212(2) (2012) 355364. https://doi.org/10.1016/j.jmatprotec.2011.09.020

[4] D. Herzog, V. Seyda, E. Wycisk and C. Emmelmann, Additive manufacturing of metals, Acta Mater. 171 (2016) 371-392. https://doi.org/10.1016/j.actamat.2016.07.019

[5] S. Bremen, W. Meiners and A. Diatlov, Selective Laser Melting A manufacturing technology for the future, Laser Tech. J. 9(2) (2012) 33-38. https://doi.org/10.1002/latj.201290018

[6] M. F. Zaeh and G. Branner, Investigations on residual stresses and deformations in selective laser melting, Prod. Eng. 4(1) (2009) 35-45. https://doi.org/10.1007/s11740-009-0192-y

[7] P. Mercelis and J.-P. Kruth, Residual stresses in selective laser sintering and selective laser melting, Rapid Prototyp. J. 12(5) (2006) 254-265.

[8] M. J. Park, H. N. Yang, D. Y. Jang, J. S. Kim and T. E. Jin, Residual stress measurement on welded specimen by neutron diffraction, J. Mater. Process. Technol. 155-156 (2004) 1171-1177. https://doi.org/10.1016/j.jmatprotec.2004.04.393

[9] C. Casavola, S. L. Campanelli and C. Pappalettere, Preliminary investigation on distribution of residual stress generated by the selective laser melting process, J. Strain Anal. Eng. Des. 44 (2009) 93-105. https://doi.org/10.1243/03093247JSA464 